\title{
Comments on gestational diabetes mellitus: from pathophysiology to clinical practice
}

\author{
Pavlos Poulakos, Gesthimani Mintziori, Efrosini Tsirou, Eleftheria Taousani, \\ Dimitra Savvaki, Vikentia Harizopoulou, Dimitrios G. Goulis
}

Unit of Reproductive Endocrinology, First Department of Obstetrics and Gynecology, Medical School, Aristotle University of Thessaloniki, Thessaloniki, Greece

\begin{abstract}
Gestational diabetes mellitus (GDM) is a topic of major interest, as it affects up to $16 \%$ of pregnant women and may lead to adverse pregnancy outcomes, which, however, are preventable by appropriate treatment. The aim of the present study was to discuss basic concepts and to critically appraise recent updates on practical issues in the field of GDM. GDM pathophysiology, long-term complications including "fetal programming" and GDM diagnosis are discussed, while clinical practice guidelines on follow-up, medical nutrition therapy, oral hypoglycemic agents and insulin treatment are also reviewed. GDM comprises a serious yet preventable public health problem and prevention by lifestyle changes, early detection and adequate treatment can lead to better health outcomes for both mothers with GDM and their offspring.
\end{abstract}

Key words: Diagnosis, Gestational diabetes, Pathophysiology, Pregnancy, Treatment

\section{INTRODUCTION}

Gestational diabetes mellitus (GDM) is a topic of major interest, for three main reasons. First, it is a very common condition concerning up to $16 \%$ of pregnant women in high-risk populations; ${ }^{1}$ second, it can lead to adverse pregnancy outcome, as it affects both the mother and her embryo/neonate; and, third, appropriate treatment is capable of both improving maternal health-related quality of life as well as reducing perinatal morbidity and mortality. ${ }^{2}$

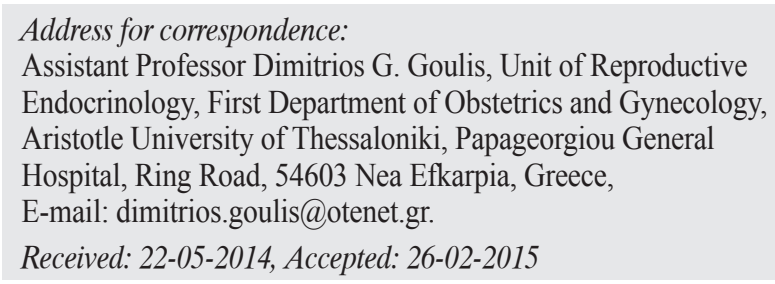

The current study aims to discuss basic concepts and to critically appraise recent updates on practical issues in the field of GDM. More specifically, seven observations will be made on pathophysiology, long-term complications, diagnosis, follow-up, medical nutrition therapy, oral hypoglycemic agents and insulin treatment.

\section{PATHOPHYSIOLOGY}

According to the World Health Organization (WHO), diabetes mellitus (DM) is classified into four broad categories: DM type 1, DM type 2, other rare types of DM and GDM. DM type 1 is characterized by autoimmune destruction of insulin-producing beta cells of the pancreas and has been linked to a genetic susceptibility, a diabetogenic trigger and/or exposure 
to a driving antigen. ${ }^{3}$ On the other hand, DM type 2 is primarily characterized by insulin resistance and relative insulin deficiency. Apart from DM type 1 and 2 , there are a large number of rare types of DM, such as genetic defects of $\beta$-cell function or insulin action, diseases of the exocrine pancreas and druginduced DM. Finally, GDM is considered a fourth, distinct type of DM, defined as glucose intolerance that appears or is recognized for the first time during pregnancy. ${ }^{4,5}$ Nevertheless, GDM is closely associated with DM type 2, as they share many key pathophysiologic characteristics and, most importantly, insulin resistance.

Figure 1 illustrates the close relationship between GDM and DM type 2. The horizontial axis represents time and the vertical insulin production and/or action. The green line represents a woman who will never develop either GDM or DM type 2 ("control"). Although the production and action of insulin declines with age, she always remains above the threshold of developing clinical DM. During pregnancy, this woman develops insulin resistance, mainly due to hormone production by the placenta that antagonizes insulin. Nevertheless, she will not develop GDM, as the pancreas has functional reserves and is able to produce larger amounts of insulin. Immediately after delivery, insulin production and action return to the pre-pregnancy level. On the other hand, the red line represents a woman who will develop GDM, and possibly DM type 2 ("patient"). She is probably genetically predisposed to DM type 2 and/or has adopted a suboptimal lifestyle (e.g. obesity, lack of exercise). Although her insulin action is already at a lower level compared to control women, she has not yet developed DM type 2, being above the relevant threshold. During pregnancy, a third factor, insulin resistance, is added to genetic predisposition and suboptimal lifestyle. As a consequence, she develops GDM, since the pancreas, although producing larger amounts of insulin, does not have adequate functional reserves to completely overcome insulin resistance. Immediately after delivery, the placenta is removed and insulin production and action return to the prepregnancy level. On an average, $90 \%$ of GDM cases are transient. ${ }^{6,7}$ The remaining $10 \%$ represents either woman who were very close to the threshold of developing DM type 2 or women who already had mild forms of DM type 2 which were not diagnosed before pregnancy. In the event of the former, it is the genetic predisposition and the suboptimal lifestyle that lead to the development of DM type 2 and not the pregnancy itself. ${ }^{6,7}$ These women with a history of GDM have up to a $60 \%$ likelihood $^{8-10}$ of developing GDM in a

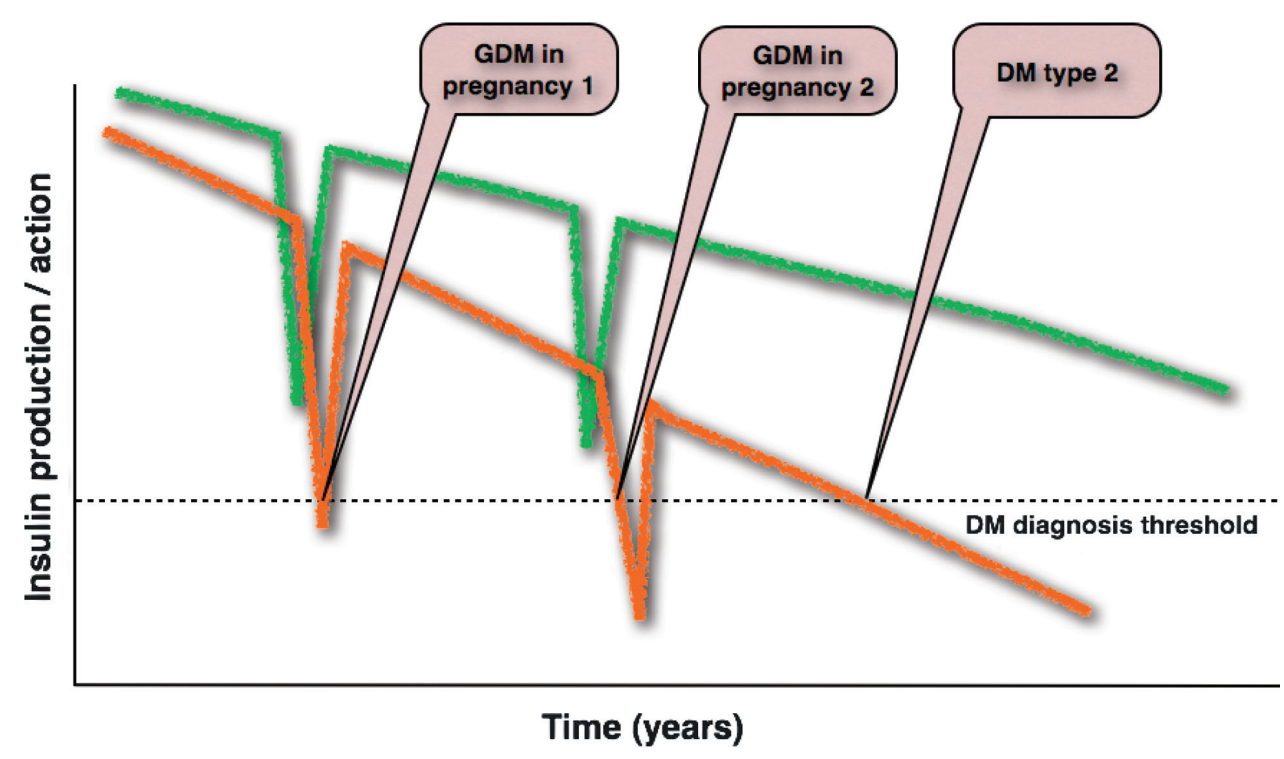

Figure 1. Production and action of insulin during the lifespan of a woman. The green line represents a woman that will never develop either gestational diabetes mellitus (GDM) or diabetes mellitus (DM) type 2 ("control"). The red line represents a woman that will develop GDM and, possibly, DM type 2 ("patient"). See text for details. 
future pregnancy. The main reason for this high probability is that, as time goes by, functional reserves of the pancreas are reduced. An additional reason could be further deterioration of lifestyle. Finally, GDM is a well-established risk factor for developing DM type 2 in the future. ${ }^{11}$ Women with a history of GDM have approximately a $30 \%$ likelihood of developing DM type 2 within 10 years ${ }^{12,13}$ Once again, the main reasons are genetic predisposition and a suboptimal lifestyle and not the pregnancies themselves. The increase of insulin resistance induced by the placenta lasts only until delivery; there is no evidence that it adds additional strain to the pancreas of a woman who is genetically or environmentally predisposed to developing DM type 2.

Figure 2 demonstrates the basic concepts of the GDM pathophysiology.

\section{LONG-TERM COMPLICATIONS}

The short-term complications for a mother with GDM include pre-eclampsia, polyhydramnios and cesarian section. Similarly, the main short-term complications for the fetus-neonate include perinatal mortality, macrosomia, obstetric trauma, hypoglycemia, hyperbilirubinemia and hypocalcemia., ${ }^{2,14}$

The "fetal origin of adult disease" concept states that adverse environmental insults during pregnancy may lead to disease later in life. ${ }^{15}$ Figure 3 illustrates this concept in the field of GDM. Let us assume that the pancreas of the embryo is capable of producing insulin at a range from 0 to 100 (arbitary units), as it has no genetic predisposition to DM. In underfeeding
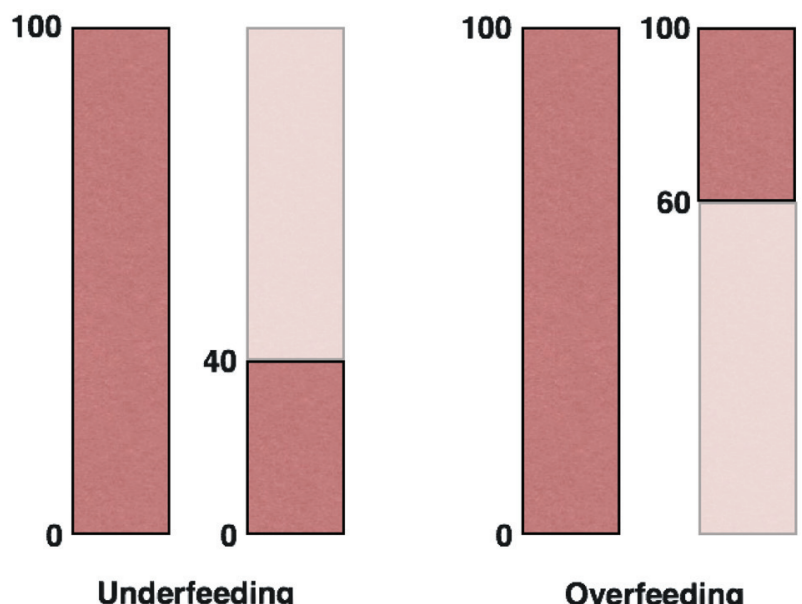

Figure 3. Fetal origin of adult disease. Under underfeeding conditions (left columns), the embryonic pancreas secretes smaller amounts of insulin (range 0 - 40) and loses permanently the capability of producing larger amounts (range 40 - 100). Under overfeeding conditions (right columns), the embryonic pancreas "learns" to secrete larger amounts of insulin (range 60 - 100). See text for details.

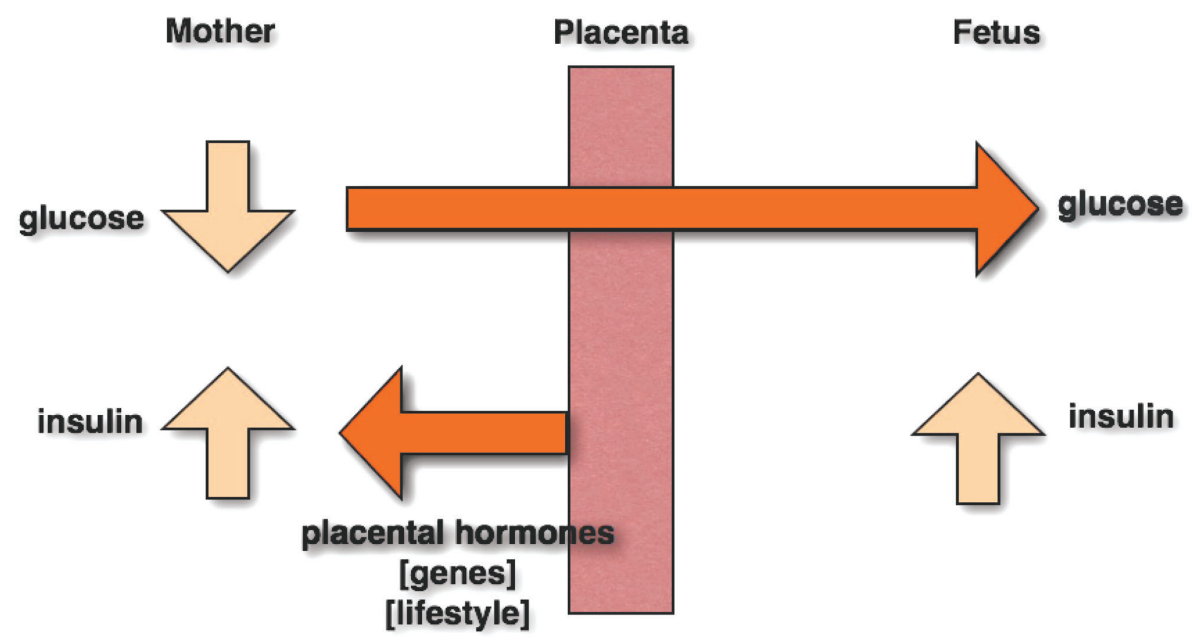

Figure 2. Pathophysiology of gestational diabetes mellitus. During pregnancy, insulin resistance is increased due to production of placental hormones that antagonize insulin action. This increase is added to the insulin resistance due to genetic susceptibility and / or suboptimal lifestyle. In the event there is adequate release of insulin by the maternal pancreas, euglycemia is preserved and the embryo is not affected. However, when insulin release is inadequate, hyperglycemia occurs (gestational diabetes mellitus) and an excess amount amount of glucose is transferred via the placenta to the embryo. Neither maternal nor embryo insulin crosses the placenta. 
conditions (e.g. starvation of the mother), the embryonic pancreas would secrete smaller amounts of insulin (e.g. at a range from 0 to 40), as these amounts will be appropriate for metabolising the reduced glucose load delivered from the mother through the placenta. Nevertheless, the pancreas, which has not completed its organogenesis, develops a certain "plasticity" and loses permanently the capability of producing larger amounts of insulin (e.g. at a range from 60 to 100). After delivery, the neonate has no obvious metabolic complications, as the reduced pancreatic reserves are still capable of fulfilling its nutritional needs, especially if he/she is in a state of underfeeding. Nevertheless, if later in life the child or the adult is exposed to increased carbohydrate intake, he/she will not have the capacity to produce appropriate amounts of insulin and, consequently, will develop DM type 2. In other words, the "fetal programming" of the pancreas for low insulin secretion, which was appropriate for the embryo, prevents adaptation to another type of diet and results in disease during childhood, adolescence or adulthood.

Examining the opposite case, in overfeeding conditions (e.g. obesity of the mother), the embryonic pancreas would secrete larger amounts of insulin (e.g. at a range from 60 to 100), as these amounts will be appropriate for metabolising the increased glucose load delivered from the mother through the placenta. Nevertheless, the pancreas, which has not completed its organogenesis, develops a certain "plasticity" and permanently produces larger amounts of insulin (e.g. at a range from 60 to 100). After delivery, the neonate has no obvious metabolic complications, as the reduced pancreatic reserves are still capable of fulfilling its nutritional needs. Nevertheless, later in life, especially if the child or the adult is exposed to increased carbohydrate intake, he/she will not have the capacity to produce appropriate amounts of insulin, as the pamcreas has been "exhausted" and, consequently, will develop DM type 2.

Figure 4 illustrates that both under- and overfeeding states during pregnancy may lead, via different pathophysiological mechanisms, to insulin deficiency and DM type 2 later in life. This "inverted-U" shape is quite common in endocrine and metabolic diseases. An example could be production of growth hormone (GH): both GH deficiency and excess (acromegaly) can result, via different mechanisms, in the same adverse outcome, namely cardiovascular disease.

It is obvious that the concept of pancreatic "plasticity" requires a pathophysiological basis. It has been suggested that a high-fat diet affects key $\beta$-cell gene and protein expression. A series of studies have shown the impact of a high-fat diet on the offspring's mRNA expression, protein concentrations of glucose transporter 2, glucokinase and Pdx-1. ${ }^{16}$ Experimental data indicate that epigenetic mechanisms may account for the association between early life nutrition

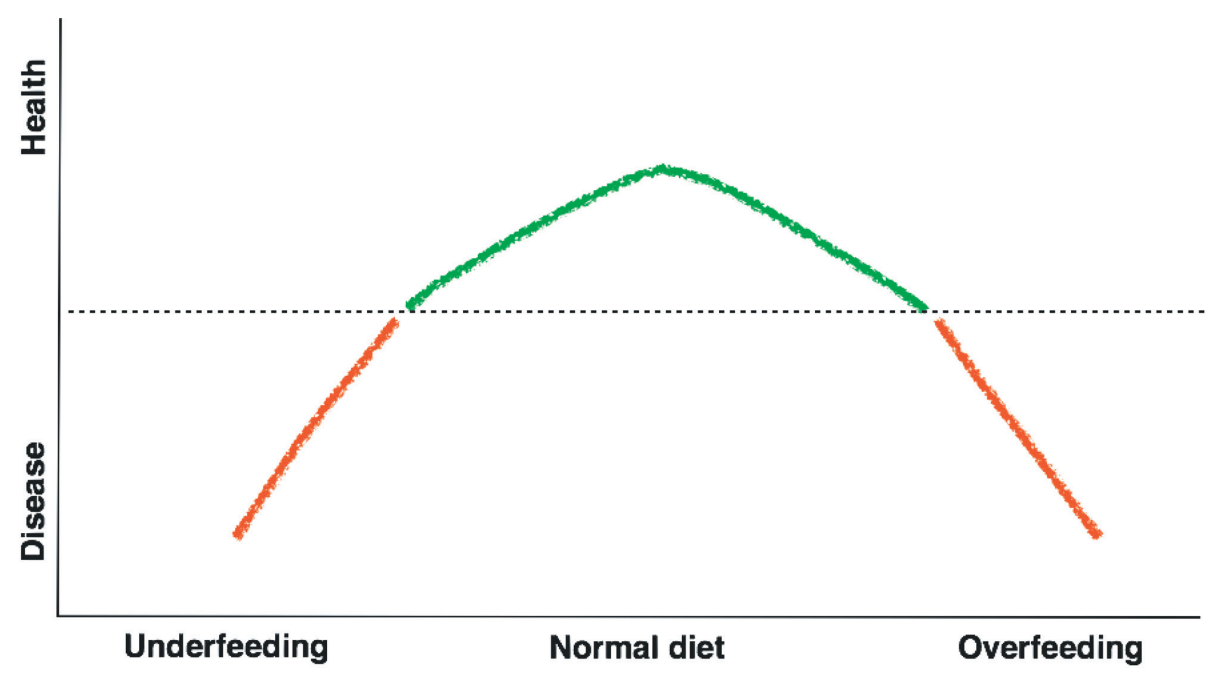

Figure 4. Fetal origin of adult disease. Both under- and over-feeding states during pregnancy may lead, via different pathophysiological mechanisms, to insulin deficiency and diabetes mellitus type 2 later in life. 
and glucose metabolism in adult life. MicroRNAs (miRNAs), which comprise a major class of small, non-coding RNAs, may play an important role in these epigenetic mechanisms. ${ }^{17}$

\section{DIAGNOSIS}

Over the last few decades, the diagnosis of GDM has been a topic of debate, as different diagnostic criteria have been applied according to different rationales..$^{5,18-20}$ All these criteria are based on fasting or non-fasting maternal glucose concentrations, most usually by means of an oral glucose tolerance test (OGTT). As the most important issue in cases of GDM is the development of embryonic, neonatal or maternal complications, the latter have to be the "gold standard" against which different sets of maternal glucose concentrations will be compared. That is to say, maternal glucose concentrations will need to be used to predict the development of complications with the highest possible sensitivity and specificity. Nevertheless, the adoption of a low glucose threshold results in overdiagnosis (Figure 5), as many women who are not likely to develop complications will be diagnosed as having GDM. In contrast, the adoption of a high threshold results in underdiagnosis, as many

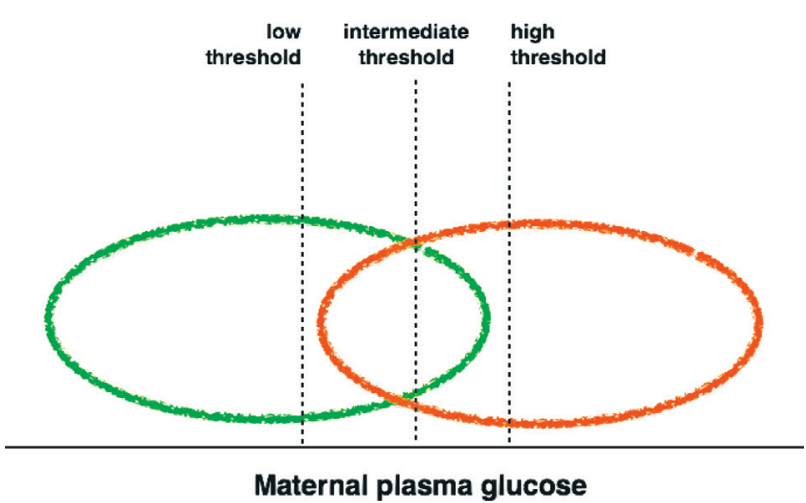

Figure 5. Diagnosis of gestational diabetes mellitus. The green ellipsoid represents women that will not develop gestational diabetes mellitus (GDM)-associated complications. The red ellipsoid represents women that will develop such complications. The adoption of a low threshold results in overdiagnosis (part of the green ellipsoid, right of the "low threshold line"). Similarly, the adoption of a high threshold results in underdiagnosis (part of the red ellipsoid, left of the "high threshold line"). Even the adoption of an intermediate threshold will result in a lower, but important, risk for over- and underdiagnosis. women who would go on to develop complications will be diagnosed as not having GDM. Even the adoption of an intermediate threshold will result in a lower but serious risk for over- and underdiagnosis. Otherwise expressed, decreasing the diagnostic threshold will result in high sensitivity but low specificity of maternal glucose concentration in the diagnosis of GDM. The opposite is true for increasing the diagnostic threshold. Thus, the definition of GDM by means of maternal glucose concentrations constitutes a difficult challenge.

The Hyperglycemia and Adverse Pregnancy Outcomes (HAPO) study attempted to tackle this challenge by assessing 25,505 pregnant women. The study pointed out that there is no specific threshold for maternal serum glucose over which the chance of developing maternal or neonatal complications increases significantly. By contrast, the correlation between the degree of glycemia and the probability of adverse outcomes (e.g. neonatal birth weight $>90^{\text {th }}$ percentile, primary cesarean section, neonatal clinical hypoglycemia, cord-blood C-peptide concentrations $>90^{\text {th }}$ percentile) is linear. ${ }^{21}$ Therefore, the threshold for GDM diagnosis had to be the product of a consensus.

Based on the data from the HAPO study, the International Association of Diabetes and Pregnancy Study Groups (IADPSG) decided to set the threshold at an odds ratio of 1.75 for developing specific complications, namely increase in neonatal fat mass, being large-for-gestational-age (LGA) and cord C-peptide concentrations $>90$ percentile, compared with mean glucose concentrations (Table 1). This odds ratio corresponds to a fasting plasma glucose of $92 \mathrm{mg} /$ $\mathrm{dl}$ or 180 and $153 \mathrm{mg} / \mathrm{dl}$ after an OGTT with $75 \mathrm{~g}$ of glucose at one and two hours, respectively. ${ }^{22}$ In other words, a diagnosis of GDM set by the IADPSG criteria means that the specific woman has a $75 \%$ increased risk (i.e. odds ratio 1.75) of developing complications as compared to a woman with mean glucose concentrations.

According to the same criteria, if a woman has a fasting glucose of more than $126 \mathrm{mg} / \mathrm{dl}$, a random glucose of more than $200 \mathrm{mg} / \mathrm{dl}$ or a glycosylated hemoglobin $\left(\mathrm{HbA}_{1 \mathrm{c}}\right)$ of more than $6.5 \%$, then a diagnosis of pre-existing (overt) DM has to be made. ${ }^{20}$ As the IADPSG criteria are based on the HAPO data, the same OGTT methodology needs to be applied. This 
Table 1. Different sets of plasma glucose concentrations as thresholds for GDM diagnosis. Plasma glucose concentrations correspond to odds ratios, as far as increase in neonatal fat mass, large-forage (LGA) and cord C-peptide concentrations $>90$ percentile are concerned, compared with mean glucose concentrations. In other words, a diagnosis of GDM set by the IADPSG criteria means that the specific woman has $75 \%$ increased risk (i.e. odds ratio 1.75 ) of developing complications, compared with a woman with mean glucose concentrations. The table illustrates the corresponding diagnostic sets of glucose concentrations, an odds ratio of 1.5 or 2 were adopted.

\begin{tabular}{cccc}
\hline & \multicolumn{3}{c}{ Odds ratio } \\
\cline { 2 - 4 } Time (min) & 1.5 & 1.75 & 2 \\
\cline { 2 - 4 } & \multicolumn{3}{c}{ Plasma glucose (mg/dl) } \\
\hline 0 & 90 & 92 & 95 \\
60 & 167 & 180 & 191 \\
120 & 142 & 153 & 162 \\
\hline
\end{tabular}

Source: Coustan DR, et al. Am J Obstet Gynecol 2010, 202:654.e1.

includes an OGTT with 75 gr of glucose and three blood samples drawn at 0,60 and 120 min. Only one abnormal value is required for the diagnosis (Table 2).

The IADPSG criteria have a number of advantages. They represent a unique opportunity for uniformity in GDM diagnosis, are simple and can be compared with similar criteria for DM diagnosis in the nonpregnant state. On the other hand, they have been developed based on observational studies of GDM complications and cannot necessarily be used as treatment thresholds. In addition, they increase the GDM prevalence to $16 \%$, being more inclusive than older criteria. ${ }^{23,24}$

Regarding screening for GDM, the latest guidelines drawn up by the Endocrine Society (ES) are the first to recommend universal testing for overt DM in early pregnancy. A fasting plasma glucose, $\mathrm{HbA}_{1 \mathrm{c}}$ or an untimed random plasma glucose at the first prenatal visit (before 13 weeks of pregnancy or

Table 2. Diagnosis of GDM according to the International Association of Diabetes and Pregnancy Study Groups (IADPSG) criteria.

\begin{tabular}{cc}
\hline Time $(\mathbf{m i n})$ & Plasma glucose $(\mathbf{m g} / \mathbf{d l})$ \\
\hline 0 & $\geqq 90(<126)$ \\
60 & $\geqq 180$ \\
120 & $\geqq 153$ \\
\hline
\end{tabular}

- Oral load 75 g glucose.

- One abnormal values is required for the diagnosis. as soon as possible thereafter) is recommended for those women not known to already have DM. In the event of overt DM, a second test (either a fasting plasma glucose, untimed random plasma glucose, $\mathrm{HbA}_{1 \mathrm{c}}$ or OGTT) is recommended on another day to confirm the diagnosis. In all pregnant women in whom no overt DM or GDM has been diagnosed, a 2-hour 75-g OGTT should be performed at 24 to 28 weeks of pregnancy. ${ }^{25}$

\section{FOLLOW-UP}

All women diagnosed with GDM should be followed up during their pregnancy. Both maternal (metabolic) and embryonic (ultrasonographic) parameters have to be applied, as they reflect different aspects of carbohydrate metabolism during pregnancy. Metabolic parameters that have been proposed for the follow-up of women with GDM are home blood glucose measurements (HBGM), continuous glucose monitoring (CGM), $\mathrm{HbA}_{1 \mathrm{c}}$, fructosamine and body weight. Glucose monitoring, by means of HBGM, needs to include measurements of morning glucose, in the fasting state, as well as post-prandial measurements, usually one hour after the meal. CGM, usually in patients with DM type 1 who are on insulin pumps, seems to provide the greatest amount of information. ${ }^{26}$ Finally, $\mathrm{HbA}_{1 \mathrm{c}}$ can provide additional information, though its use in pregnancy is limited..$^{27-29}$

Ultrasonographic parameters that have been proposed for the follow-up of women with GDM are estimated fetal weight (EFW), head circumference (HC), abdominal circumference (AC), femoral length (FL) and presence of polyhyramnios. Fetal growth ultrasound during the second and third trimesters of pregnancy seems to be the most important clinical assessment tool. ${ }^{30}$

Practical suggestions for HBGM during pregnancy include:

- Glucose must be checked each morning in the fasting state, as well as 1-h after each main meal.

- Patient-specific targets must be set.

- Women with GDM have to retain fasting glucose concentrations between 65 and $95 \mathrm{mg} / \mathrm{dl}$ and 1-h postprandial $<140 \mathrm{mg} / \mathrm{dl}$. 
- There is no need for routine evaluation of $\mathrm{HbA}_{1 \mathrm{c}}$ during the second and third trimesters of pregnancy.

\section{MEDICAL NUTRITION THERAPY}

There is evidence that effective treatment that leads to normalization of maternal glucose concentrations results in a better pregnancy outcome. ${ }^{2,31}$ The cornerstones of GDM treatment are lifestyle modifications (medical nutrition therapy, physical exercise, cessation of smoking) and insulin administration. Oral hypoglycemic agents seem to play an important role, which is constantly expanding.

Optimal body weight before pregnancy and its maintenance throughout this period has a significant impact on maternal and neonatal health. There is evidence that neonates of overweight and obese mothers have an elevated risk for perinatal morbidity and mortality compared to neonates of mothers of normal weight. ${ }^{32}$ The main principles of diet in women whose pregnancies are complicated by GDM are the involvement of a multi-disciplinary team (including endocrinologist, obstetrician-gynecologist, dietician, ergophysiologist, diabetes nurse) and the construction of a dietary program that restores euglycemia, prevents ketosis and leads to optimal weight gain, taking into consideration all possible effects on the health of the embryo.

Various guidelines have been developed to minimize the negative impact of either inadequate or excessive weight gain during pregnancy. A very important goal is to achieve optimal weight before pregnancy. In those instances where this is not the case, the Institute of Medicine (IOM) has developed weight gain recommendations for pregnant women based on their pre-pregnancy body mass index (BMI) (Table 3). ${ }^{33}$ These recommendations deal with both total weight gain and rate of gain during pregnancy.

Important issues remain to be solved. There is no clear evidence of the need for possible diet modifications according to maternal race and age. Obviously, multiple pregnancy should be taken into account when setting up a diet plan. The total amount of caloric intake should be calculated in a uniform way according to the pre-pregnancy and current BMI. Other unresolved issues are the distribution of calories in the meals and the percentage of carbohydrate intake. Weight loss during pregnancy is a much debated topic. ${ }^{34}$

\section{ORAL HYPOGLYCEMIC AGENTS}

In contrast to other oral hypoglycemic agents, glibenclamide (gliburide in the US) and metformin have been proposed for the treatment of GDM.

\section{Glibenclamide}

Glibenclamide most probably does not cross the placenta. Its use during pregnancy has not been associated with neonatal hypoglycemia or increase in congenital anomalies. According to data from randomized control trials (RCTs), glibenclamide is comparable to insulin in terms of effectiveness, though an increase in macrosomia has been reported. ${ }^{35,36}$

\section{Metformin}

Metformin readily crosses the placenta. Nevertheless, its use during pregnancy has not been associated with an increase in congenital anomalies. ${ }^{37}$ According to data from RCTs, metformin is comparable to insulin in terms of effectiveness, though in $50 \%$ of cases insulin has to be added. ${ }^{38}$ A possible indication for metformin

Table 3. Recommendations for total and rate of weight gain during pregnancy by pre-pregnancy body mas index (BMI). Source: Kathleen M, et al, Institute of Medicine, National Research Council, 2009

\begin{tabular}{lccccc}
\hline \multirow{2}{*}{ Prepregnancy BMI } & \multicolumn{2}{c}{ Total weight gain } & & \multicolumn{2}{c}{ Rates of weight gain* 2nd and 3rd trimester } \\
\cline { 2 - 3 } \cline { 5 - 6 } & Range in kg & Range in lbs & & Mean (range) in kg/week & Mean (range) in lbs/week \\
\hline Underweight $\left(<18.5 \mathrm{~kg} / \mathrm{m}^{2}\right)$ & $12.5-18$ & $28-40$ & & $0.51(0.44-0.58)$ & $1(1-1.3)$ \\
Normal weight $\left(18.5-24.9 \mathrm{~kg} / \mathrm{m}^{2}\right)$ & $11.5-16$ & $25-35$ & & $0.42(0.35-0.50)$ & $1(0.8-1)$ \\
Overweight $\left(25.0-29.9 \mathrm{~kg} / \mathrm{m}^{2}\right)$ & $7-11.5$ & $15-25$ & & $0.28(0.23-0.33)$ & $0.6(0.5-0.7$ \\
Obese $\left(\geq 30.0 \mathrm{~kg} / \mathrm{m}^{2}\right)$ & $5-9$ & $11-20$ & & $0.22(0.17-0.27)$ & $0.5(0.4-0.6)$ \\
\hline
\end{tabular}

*Calculations assume a 0.5-2 kg (1.1-4.4 lbs) weight gain in the first trimester (based on Siega-Riz et al, 1994; Abrams et al, 1995; Carmichael et al, 1997). 
use during the first trimester of pregnancy could be the prevention of GDM in women with polycystic ovary syndrome (PCOS). However, a recent study failed to show such an effect. ${ }^{39} \mathrm{~A}$ recent meta-analysis by our group concluded that the existing evidence supports the efficacy and safety of metformin during pregnancy with respect to pregnancy outcomes. ${ }^{40}$

\section{INSULIN}

Insulin treatment remains the cornerstone of GDM treatment. The main clinical questions are the same, as in any other case of insulin usage:

- When (must insulin be started)?

- Which (type of insulin needs to be used)?

- How (will insulin be administered: regime, dosage)?

- What (are the therapeutic targets)?

The follow-up parameters that need to be taken into consideration for insulin initiation in a woman whose pregnancy has been complicated by GDM are both metabolic (HBGM, CGM, HbA 1 , body weight) and ultrasonographic (EFW, HC, AC, FL, polyhydramnios).

The rapid-acting insulin analogues lispro and aspart, like any other type of insulin, do not cross the placenta. There is no evidence of increased risk for congenital anomalies or antibody production. They both comprise safe therapeutic options, with similar efficacy, in terms of metabolic control and number of hypoglycemic episodes..$^{41,42} \mathrm{An}$ intermediate-acting insulin [neutral protamine Hagedorn (NPH) or analogue] before bed is an obvious option for fasting hyperglycemia, whereas a rapid- or ultra-rapid-acting insulin before a specific meal has to be considered to deal with post-prandial hyperglycemia.

Recently, an RCT assessed pregnant women with DM type 1 treated with either the intermediate-acting insulin analogue detemir $(\mathrm{n}=152)$ or NPH $(\mathrm{n}=$ 158)..$^{43}$ The main study outcome was the achievement of glycemic control, assessed by $\mathrm{HbA}_{1 \mathrm{c}}$, during gestational week 36 ; secondary outcomes were the effectiveness and safety of insulin detemir in terms of pregnancy outcome. Insulin detemir achieved comparable glycemic control, levels of $\mathrm{HbA}_{1 \mathrm{c}}$ and number of hypoglycemic episodes compared with insulin NPH. Insulin detemir resulted in significantly decreased fasting glucose and comparable pregnancy outcome. ${ }^{43}$ According to the recent Endocrine Society guidelines ${ }^{25}$ insulin detemir may be initiated during pregnancy whenever it is suspected that NPH insulin might result in hypoglycemia. Women taking insulin detemir or insulin glargine before pregnancy may continue their use throughout pregnancy.

\section{CONCLUSIONS}

On the basis of the basic concepts discussed above and a number of recent updates that have been appraised regarding practical issues in the field of GDM, it can be concluded that:

- GDM is closely associated with DM type 2, as they share key pathophysiologic characteristics, mainly with regard to insulin resistance. A history of GDM is a risk factor for developing GDM in subsequent pregnancies, as well as DM type 2, in later life.

- The "fetal programming" of the pancreas for low insulin secretion, although appropriate for the embryo, prevents adaptation to another type of diet and may result in glucose intolerance during childhood, adolescence or adulthood. In a analogous though opposite way, the "fetal programming" for high insulin secretion may exhaust the pancreas and result in glucose intolerance in later life (fetal origin of dult disease).

- According to the IADPSG criteria, the diagnosis of GDM is established if fasting plasma glucose is greater than $92 \mathrm{mg} / \mathrm{dl}$ or greater than 180 and $153 \mathrm{mg} / \mathrm{dl}$ at one and two hours, respectively, after a 2-hour 75-g OGTT.

- The follow-up of a woman with GDM must include both metabolic (HBGM, CGM, $\mathrm{HbA}_{1 \mathrm{c}}$, fructosamine, body weight) and ultrasonographic parameters (EFW, HC, AC, FL, polyhydramnios).

- The main principles of diet in women whose pregnancies are complicated by GDM are the involvement of a multi-disciplinary team and the construction of a dietary program that restores euglycemia, prevents ketosis and leads to optimal weight gain, according to the IOM recommendations. 
- Oral hypoglycemic agents, specifically glipuride, glibenclamide and metformin, have been proposed for the treatment of GDM. Metformin seems to constitute an efficient and safe treatment alternative that can be used in selected cases.

- Insulin treatment remains the cornerstone of GDM treatment. The rapid-acting insulin analogues lispro and aspart, as well as the intermediate-acting insulin analogue detemir, are effective and safe alternatives to human insulin.

\section{REFERENCES}

1. Halperin IJ and Feig DS, 2014 The role of lifestyle interventions in the prevention of gestational diabetes. Curr Diab Rep 14: 452.

2. Crowther CA, Hiller JE, Moss JR, McPhee AJ, Jeffries WS, Robinson JS, 2005 Effect of treatment of gestational diabetes mellitus on pregnancy outcomes. N Engl J Med 352: 2477-2486.

3. Bluestone JA, Herold K, Eisenbarth G, 2010 Genetics, pathogenesis and clinical interventions in type 1 diabetes. Nature 464: 1293-1300.

4. American Diabetes Association, 2011 Standards of medical care in diabetes-2011. Diabetes Care 34: Suppl 1: 11-61.

5. Metzger BE, Buchanan TA, Coustan DR, et al, 2007 Summary and recommendations of the Fifth International Workshop-Conference on Gestational Diabetes Mellitus. Diabetes Care 30: Suppl 2: 251-260.

6. Catalano PM, Vargo KM, Bernstein IM, Amini SB, 1991 Incidence and risk factors associated with abnormal postpartum glucose tolerance in women with gestational diabetes. Am J Obstet Gynecol 165: 914-919.

7. Kjos SL, Buchanan TA, Greenspoon JS, Montoro M, Bernstein GS, Mestman JH, 1990 Gestational diabetes mellitus: the prevalence of glucose intolerance and diabetes mellitus in the first two months post partum. Am J Obstet Gynecol 163: 93-98.

8. Getahun D, Fassett MJ, Jacobsen SJ, 2010 Gestational diabetes: risk of recurrence in subsequent pregnancies. Am J Obstet Gynecol 203: 467.e1-6.

9. MacNeill S, Dodds L, Hamilton DC, Armson BA, VandenHof M, 2001 Rates and risk factors for recurrence of gestational diabetes. Diabetes Care 24: 659-662.

10. Moses RG, 1996 The recurrence rate of gestational diabetes in subsequent pregnancies. Diabetes Care 19: 1348-1350.

11. Feig DS, Zinman B, Wang X, Hux JE, 2008 Risk of development of diabetes mellitus after diagnosis of gestational diabetes. CMAJ 179: 229-234

12. Kaaja RJ, Greer IA, 2005 Manifestations of chronic disease during pregnancy. JAMA 294: 2751-2757.
13. Kintiraki E, Goulis DG, Mameletzi S, et al, 2013 Large- and small-for-gestational-age neonates born by women with gestational diabetes mellitus diagnosed by the new IADPSG criteria: a case-control study of 289 patients and 1108 controls. Exp Clin Endocrinol Diabetes 121: 262-265.

14. Dodd JM, Crowther CA, Antoniou G, Baghurst P, Robinson JS, 2007 Screening for gestational diabetes: the effect of varying blood glucose definitions in the prediction of adverse maternal and infant health outcomes. Aust N Z J Obstet Gynaecol 47: 307-312.

15. Parlee SD, Macdougald OA, 2014 Maternal nutrition and risk of obesity in offspring: The Trojan horse of developmental plasticity. Biochim Biophys Acta 1842: 495-506.

16. Nielsen JH, Haase TN, Jaksch C, et al, 2014 Impact of fetal and neonatal environment on beta cell function and development of diabetes. Acta Obstet Gynecol Scand 93: 1109-1122.

17. Xiao X, Zheng J, 2014 MicroRNAs: crucial modulators of fetal epigenetic programming in nutrition and glucose metabolism. Chin Med J (Engl) 127: 3521-3523.

18. Metzger BE, Gabbe SG, Persson B, et al, 2010 International association of diabetes and pregnancy study groups recommendations on the diagnosis and classification of hyperglycemia in pregnancy. Diabetes Care 33: 676-682.

19. Metzger BE, Gabbe SG, Persson B, et al, 2012 The diagnosis of gestational diabetes mellitus: new paradigms or status quo? J Matern Fetal Neonatal Med 25: 2564-2569.

20. American Diabetes Association, 2011 Diagnosis and classification of diabetes mellitus. Diabetes Care 34: Suppl 1: 62-69.

21. Metzger BE, Lowe LP, Dyer AR, et al, 2008 Hyperglycemia and adverse pregnancy outcomes. N Engl J Med 358: 1991-2002.

22. Coustan DR, Lowe LP, Metzger BE, Dyer AR, 2010 The Hyperglycemia and Adverse Pregnancy Outcome (HAPO) study: paving the way for new diagnostic criteria for gestational diabetes mellitus. Am J Obstet Gynecol 202: 654-656.

23. Claesson R, Ekelund M, Berntorp K, 2013 The potential impact of new diagnostic criteria on the frequency of gestational diabetes mellitus in Sweden. Acta Obstet Gynecol Scand 92: 1223-1226.

24. Shang M, Lin L, Ma L, Yin L, 2014 Investigation on the suitability of the International Association of Diabetes and Pregnancy Study Group diagnostic criteria for gestational diabetes mellitus in China. J Obstet Gynaecol 34: 141-145.

25. Blumer I, Hadar E, Hadden DR, et al, 2013 Diabetes and pregnancy: an endocrine society clinical practice guideline. J Clin Endocrinol Metab 98: 4227-4249.

26. de Veciana M, Major CA, Morgan MA, et al, 1995 Postprandial versus preprandial blood glucose monitoring 
in women with gestational diabetes mellitus requiring insulin therapy. N Engl J Med 333: 1237-1241.

27. Griffiths RJ, Vinall PS, Stickland MH, Wales JK, 1987 Haemoglobin A1c levels in normal and diabetic pregnancies. Eur J Obstet Gynecol Reprod Biol 24: 195-200.

28. Lurie S, Mamet Y, 2000 Red blood cell survival and kinetics during pregnancy. Eur J Obstet Gynecol Reprod Biol 93: 185-192.

29. Jovanovic L, Savas H, Mehta M, Trujillo A, Pettitt DJ, 2011 Frequent monitoring of A1C during pregnancy as a treatment tool to guide therapy. Diabetes Care 34: 53-54.

30. Balsells M, Garcia-Patterson A, Gich I, Corcoy R, 2014 Ultrasound-guided compared to conventional treatment in gestational diabetes leads to improved birthweight but more insulin treatment: systematic review and meta-analysis. Acta Obstet Gynecol Scand 93: $144-151$

31. Landon MB, Spong CY, Thom E, et al, 2009 A multicenter, randomized trial of treatment for mild gestational diabetes. N Engl J Med 361: 1339-1348.

32. Salihu HM, Alio AP, Wilson RE, Sharma PP, Kirby RS, Alexander GR, 2008 Obesity and extreme obesity: new insights into the black-white disparity in neonatal mortality. Obstet Gynecol 111: 1410-1416.

33. Institute of Medicine (US) and National Research Council (US) Committee to Reexamine IOM Pregnancy Weight Guidelines; Rasmussen KM, Yaktinen AL (eds) (2009) Weight Gain During Pregnancy: Reexamining the Guidelines. Washington (DC): National Academies Press (US); 2009.

34. Blomberg M, 2011 Maternal and neonatal outcomes among obese women with weight gain below the new Institute of Medicine recommendations. Obstet Gynecol 117: 1065-1070.

35. Lain KY, Garabedian MJ, Daftary A, Jeyabalan A,
2009 Neonatal adiposity following maternal treatment of gestational diabetes with glyburide compared with insulin. Am J Obstet Gynecol 200: 501-506.

36. Langer O, Conway DL, Berkus MD, Xenakis EM, Gonzales O, 2000 A comparison of glyburide and insulin in women with gestational diabetes mellitus. N Engl J Med 343: 1134-1138.

37. Gilbert C, Valois M, Koren G, 2006 Pregnancy outcome after first-trimester exposure to metformin: a meta-analysis. Fertil Steril 86: 658-663.

38. Rowan JA, Hague WM, Gao W, Battin MR, Moore MP, 2008 Metformin versus insulin for the treatment of gestational diabetes. N Engl J Med 358: 2003-2015.

39. Vanky E, Stridsklev S, Heimstad R, et al, 2010 Metformin versus placebo from first trimester to delivery in polycystic ovary syndrome: a randomized, controlled multicenter study. J Clin Endocrinol Metab 95: E448-E455.

40. Lautatzis ME, Goulis DG, Vrontakis M, 2013 Efficacy and safety of metformin during pregnancy in women with gestational diabetes mellitus or polycystic ovary syndrome: a systematic review. Metabolism 62: 15221534.

41. Mathiesen ER, Kinsley B, Amiel SA, et al, 2007 Maternal glycemic control and hypoglycemia in type 1 diabetic pregnancy: a randomized trial of insulin aspart versus human insulin in 322 pregnant women. Diabetes Care 30: 771-776.

42. Wyatt JW, Frias JL, Hoyme HE, et al, 2005 Congenital anomaly rate in offspring of mothers with diabetes treated with insulin lispro during pregnancy. Diabet Med 22: 803-807.

43. Mathiesen ER, Damm P, Jovanovic L, et al, 2011 Basal insulin analogues in diabetic pregnancy: a literature review and baseline results of a randomised, controlled trial in type 1 diabetes. Diabetes Metab Res Rev 27: 543-551. 